\title{
Risques oculaires du rayonnement bleu
}

\author{
J.-P. CÉSARINI ${ }^{1}$
}

(Manuscrit reçu le 11 mai 2009, accepté le 21 août 2009)

RÉSUMÉ La portion bleue $(400-500 \mathrm{~nm})$ du rayonnement visible stimule spécifiquement certains cônes et bâtonnets de la rétine. $L$ 'énergie véhiculée par ces longueurs d'onde est transférée par absorption aux pigments spécifiques. Cette énergie est suffisante pour produire des radicaux libres et de l'oxygène singulet. Des sources intenses riches en rayonnement bleu peuvent induire au niveau de la rétine des lésions soit limitées et passagères phototoxiques, soit pour de plus fortes énergies, des lésions thermiques plus ou moins définitives. Les lésions phototoxiques, par leur répétition pourraient faire le lit de la dégénérescence maculaire liée à l'âge (DMLA). Dès lors, il convient d'attirer l'attention sur les risques potentiellement liés aux éclairages modernes tels que lampes dites «lumière du jour», les lampes compactes fluorescentes économisant l'énergie (CFLs) et les diodes émettrices de lumière (LEDs) pour lesquelles une vigilance spécifique s'impose.

ABSTRACT Blue light hazards for ocular lesions.

The blue light range $(400-500 \mathrm{~nm})$ of visible radiation stimulates specifically cones and rods of the retina. The carried energy by these wavelengths is absorbed and transferred to specific pigments. Their energy is sufficient to produce free radicals and singulet form of oxygen. Intense sources, rich in blue light radiation, may induce, in the retina, phototoxic lesions either limited or short-lived or photothermal lesions more or less definitive. Repeated phototoxic lesions should be the root for the age-related maculopathy (ARM) also called late macular degeneration (AMD). As a consequence, the attention should be drawn on the potential risk linked to modern lighting as "daylight" lamp, compact fluorescent lamps, energy saving (CFLs) and light-emitting diodes (LEDs) for which a specific vigilance should be enforced.

Keywords: Ocular lesion / blue light

\section{Introduction}

Les lésions oculaires causées par la portion bleue du rayonnement visible connaissent depuis quelques années un regain d'intérêt du fait de l'introduction de nouvelles technologies liées à la lumière telles que les diodes émettrices de lumière (LED), les éclairages fluorescents compactes (CFL), la prise de conscience de l'incidence importante dans la population de la dégénérescence maculaire liée à l'âge (DMLA) qui accompagne l'allongement de la durée de vie et l'augmentation des expositions de plein air, fait sociétal avéré.

1 INSERM, Fondation Ophtalmologique A. de Rothschild, 25 rue Manin, 75019 Paris ; CES « Agents physique » AFSSET, France. 
Si les risques oculaires liés à l'éclairage artificiel et aux technologies industrielles semblent bien maîtrisés dans le domaine du travail grâce à la mise en pratique de normes internationales et européennes, il n'en est sûrement pas de même pour les risques encourus par la population en général.

De plus, l'utilisation thérapeutique du rayonnement bleu, dans les premiers jours de l'existence, pour traiter la jaunisse des nouveau-nés ou plus tard en lumino thérapie, pour traiter la dépression saisonnière, est en constante augmentation, justifiant une vigilance spécifique, en particulier, un contrôle strict des spectres d'émission.

\section{Physique des sources de rayonnement bleu}

Le rayonnement bleu est situé dans le spectre visible entre le rayonnement violet (380-420 nm) et le rayonnement vert (520-540 nm). Il est bien entendu présent dans le rayonnement solaire. Il est faiblement absorbé par l'atmosphère et apparaît à la surface de la terre dès que le disque solaire s'élève de $20^{\circ}$ environ au-dessus de l'horizon. L'irradiance spectrale du rayonnement bleu, lorsque le soleil est proche du zénith, est comprise entre 10 et $100 \mu \mathrm{Watts} / \mathrm{cm}^{2} / \mathrm{nm}$. La traversée de l'atmosphère absorbe un peu moins de la moitié du rayonnement bleu extraterrestre. Comme le montre la figure 1, le niveau énergétique du rayonnement bleu solaire est plus important que le rayonnement ultraviolet et le reste du rayonnement visible. Dans le rayonnement solaire, les quantités du rayonnement bleu qui atteignent la peau et l'œil ne sont donc pas négligeables pendant une large portion de la journée. La durée et l'intensité de cette exposition sont soumises aux fluctuations du rayonnement solaire à la surface terrestre et sont fonction de la saison, de la latitude, de l'altitude et de l'heure de la journée. D'autres paramètres influencent leur intensité tels que la qualité et la quantité de l'ennuagement et le degré d'aérosol.

Tout à fait différent est le rayonnement bleu fourni par les sources artificielles (AFE, 1993) dans les éclairages public et domestique et en général, toutes sources présentes dans l'environnement, produites par différentes technologies : laser, LED, CFL... puisque c'est volontairement que la part du rayonnement bleu est parfois augmentée afin d'obtenir un éclairage dit « naturel », type lumière du jour. Enfin, des sources intenses de rayonnement bleu sont présentes dans l'environnement industriel : soudure à l'arc, techniques de découpe, imprimerie, etc. et utilisées en thérapeutique médicale : traitement de la jaunisse néonatale, photothérapie de la dépression saisonnière, réduction du décalage horaire des voyageurs intercontinentaux, etc. 




Figure 1 - Spectre solaire parvenant à la surface de la terre, au niveau de la mer. Le pic énergétique du rayonnement solaire coüncide avec le rayonnement bleu (500 $\mathrm{nm})$.

Solar spectrum on the earth surface at sea level. The peak of energy of the solar spectrum coincides with the blue radiation.

\section{Anatomie de l'œil et physiopathologie du rayonnement bleu}

La lumière visible, transmise par la cornée, les espaces aqueux et le cristallin, réagit avec les photorécepteurs de la rétine pour produire un signal électrique nerveux communiquant le message visuel au cortex par le nerf optique et les voies optiques intracreaniennes. La rétine est constituée d'un groupe de cellules qui sont fortement oxygénées et par conséquent, susceptibles de subir des dommages par les espèces réactives de l'oxygène générés par les rayonnements UVA et bleu. Les effets de ce rayonnement ont fait l'objet de recherches spécifiques (Cronley-Dillon et al., 1986 ; Marshall, 1985 ; Young, 1991). Ces travaux ont permis de mettre en évidence la transmission du rayonnement UVA et bleu (Fig. 2). Le pigment visuel, la rhodopsine, le photopigment des bâtonnets qui présente un pic d'absorption à $500 \mathrm{~nm}$ et les pigments des cellules cônes, bleus, verts, et rouges, ont une absorption dans le rayonnement visible autour de 440,535 , et $570 \mathrm{~nm}$ respectivement.

Le spectre d'action de ce processus, dans l'environnement quotidien de la lumière du jour, est représenté par la courbe d'efficacité phototopique de la CIE (AFE, 1993). Par absorption des différentes structures de l'œil, l'énergie véhiculée 

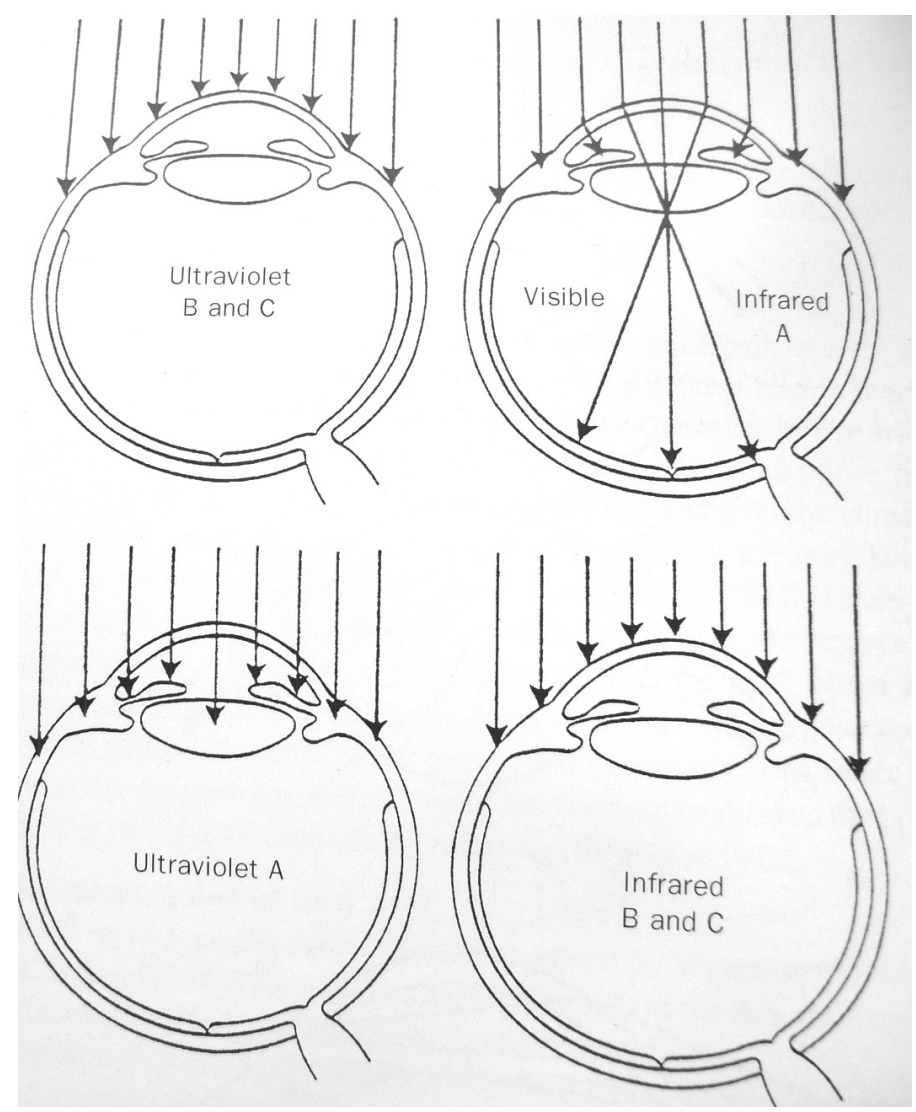

Figure 2 - Transmission et absorption sélectives des différents rayonnements par les structures oculaires. Selective absorption and transmission of the radiation by different ocular structures.

par le rayonnement bleu est libérée et transférée aux molécules susceptibles de l'absorber telles les pigments jaunes du cristallin qui apparaissent progressivement au cours du vieillissement, la rhodopsine au niveau de la rétine, la lipofuscine, et les pigments maculaires (lutéine, zéaxanthine, mésozéaxanthine), les mélanines, ou toute autre substance présente dans l'œil. En général, en plus du stimulus visuel, une irradiation optique intense peut induire des lésions photochimique ou photothermique si les niveaux d'éclairement énergétique rétiniens sont suffisamment élevés ; l'énergie en excès est transformée en chaleur et radicaux libres. 


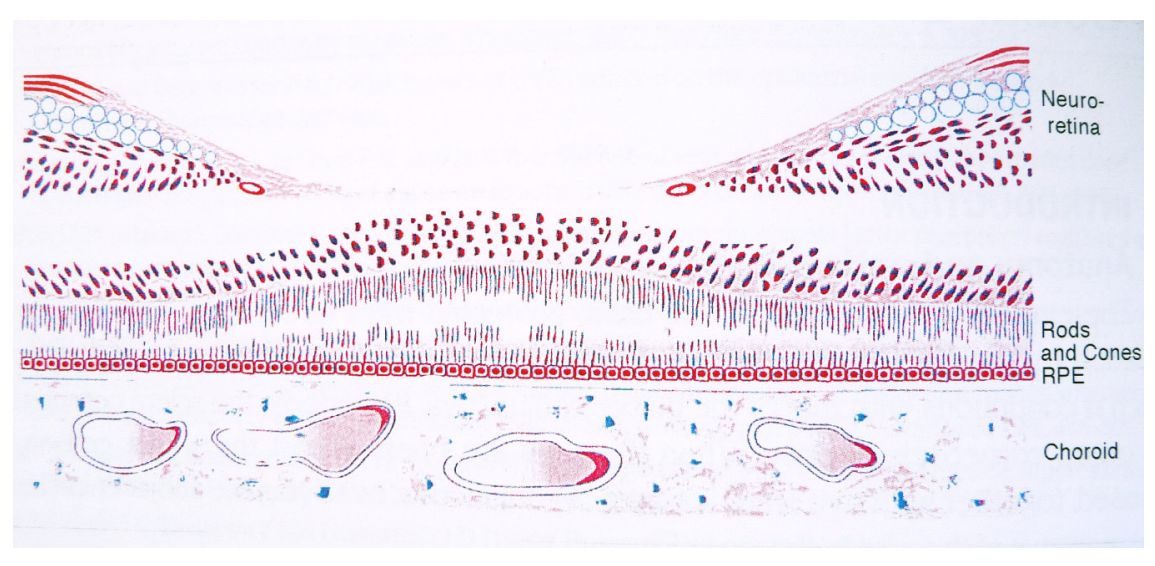

Figure 3-Histologie de la fovéa, zone la plus sensible de la rétine, où se concentre la stimulation lumineuse.

Histology of the fovea, a sensitive area of the retina, where the light is concentrated.

La zone la plus riche en récepteurs de la lumière visible (cônes et bâtonnets) est la fovéa (Fig. 3), petite surface de quelques millimètres carrés où parvient une image focalisée par le cristallin. Cette surface est le site des blessures rétiniennes.

En général, il y a cinq types de risques pathologiques liés aux radiations optiques et toute source de lumière doit être évaluée pour son risque potentiel :

(1) photokératoconjonctivite: lésion photochimique de la cornée et de la conjonctive (généralement rayonnement UV) ;

(2) lésion du cristallin par le rayonnement UV aboutissant, à long terme, à une cataracte ;

(3) blessure (thermique) par le rayonnement infrarouge de la cornée et du cristallin ;

(4) brûlure rétinienne résultant de l'exposition à une lumière visible très intense ou à une source de rayonnement infrarouge ;

(5) photorétinite : phototoxicité liée à l'agression photochimique du rayonnement bleu.

Une blessure thermique rétinienne (brûlure rétinienne accidentelle ou photocoagulation à visée thérapeutique) est produite quand les images observées par l'œil proviennent de sources lumineuses extrêmement brillantes, pulsées telle que l'émission d'un laser. Comparées aux blessures photochimiques de la rétine, des irradiances encore plus élevées sont nécessaires pour produire des brûlures thermiques. Quand l'éclairement énergétique entraîne une élévation de plus de $10^{\circ} \mathrm{C}$, 
l'examen histologique, quelques jours après l'exposition, montre des dommages structurels de l'épithélium pigmentaire avec un maximum au centre de la lésion (Fig. 4).

La blessure photochimique est produite par des expositions de durées plus longues à des niveaux d'éclairement énergétique relativement plus faibles que ceux qui sont nécessaires pour induire une blessure thermique. Elle s'accompagne en général d'une élévation thermique inférieure à $1{ }^{\circ} \mathrm{C}$. Ce type de lésion apparaît pour une dose seuil d'exposition de l'ordre de $22 \mathrm{~J} \mathrm{~cm}^{-2}$ à $446 \mathrm{~nm}$ et est indépendant de la taille du spot rétinien. On observe des dommages à travers toute la lésion qui présente une bordure nette entre les cellules pigmentées atteintes ou intactes (Fig. 5). Les atteintes structurelles touchent autant le pigment mélanique de l'épithélium pigmentaire que les pigments des photorécepteurs. Les lésions induites par la lumière bleue ressemblent à la rétinite solaire des patients ayant regardé le soleil directement. Des dommages photochimiques sont également observés dans les bâtonnets, résultant de l'absorption de la rhodopsine.

Les mécanismes de la blessure photochimique (phototoxicité) sont de deux ordres et produisent deux types différents de lésions selon :

(a) une faible irradiance rétinienne est accompagnée d'une durée d'exposition de plusieurs heures (expositions généralement répétées plusieurs jours de suite) ;

(b) une irradiance rétinienne élevée pendant une durée d'exposition courte (généralement une exposition durant quelques minutes à quelques heures).

Le spectre d'action pour produire le type (a) coïncide habituellement avec les propriétés d'absorption spectrale de la rhodopsine (pigment visuel des photorécepteurs en bâtonnets). On estime à juste titre que ce type de lésion résulte de l'absorption directe du flux lumineux par la rhodopsine (Fig. 6).

Le spectre d'action pour la réaction de type (b) présente un pic maximum dans des régions plus courtes jusque dans la région du rayonnement UV. Les experts discutent pour savoir s'il y a plusieurs spectres d'action pour la photorétinite photochimique. D'aucuns pensent qu'il n'y a qu'un spectre d'action dont le pic maximum est dans la région de l'ultraviolet dans l'œil aphake (œil sans cristallin). Cependant, en raison de l'absorption importante par le cristallin dans la région de l'UV 300 à $400 \mathrm{~nm}$, une faible fraction de l'UVA (moins de 10\%) peut atteindre la rétine et dans l'œil normal, le spectre d'action de la photorétinite présente un pic dans la région $440-450 \mathrm{~nm}$. C'est à ce mécanisme de lésions que se réfère le risque lié à la lumière bleue, «Blue-Light photochemical retinal Hazard» ou BLH. Le chromophore ou le pigment réacteur responsable du BLH n'est pas connu avec précision. On a évoqué la mélanine, le cytochrome $\mathrm{C}$ ou la riboflavine des 


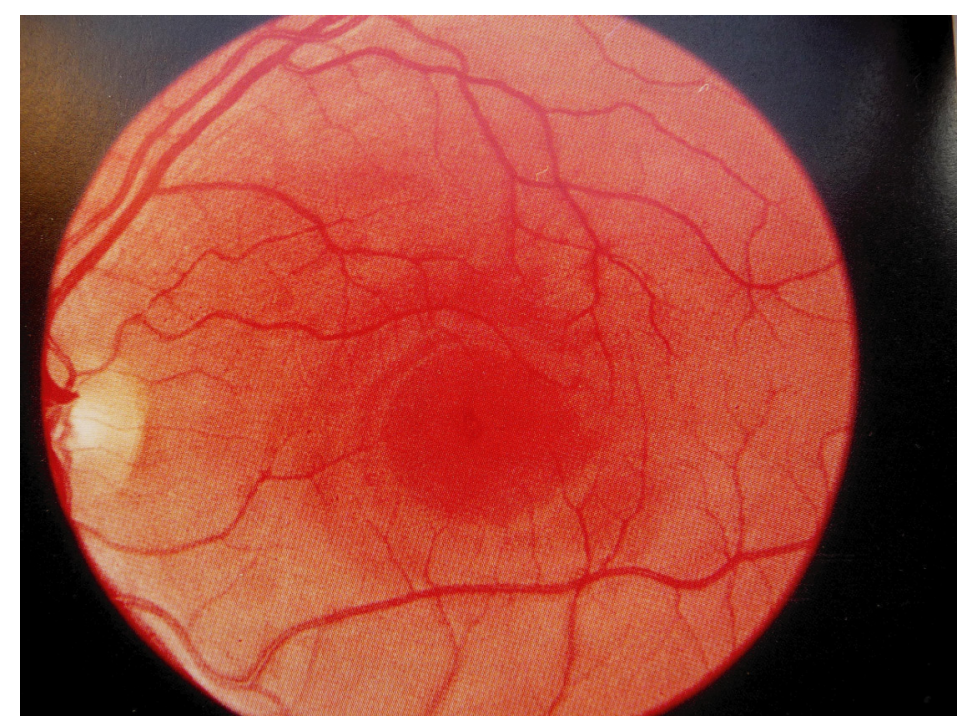

Figure 4 - Aspect de brûlure rétinienne par photo-coagulation. Ces lésions sont peu réversibles. Retinal burn by photo-coagulation. These lesions are usually not reversible.

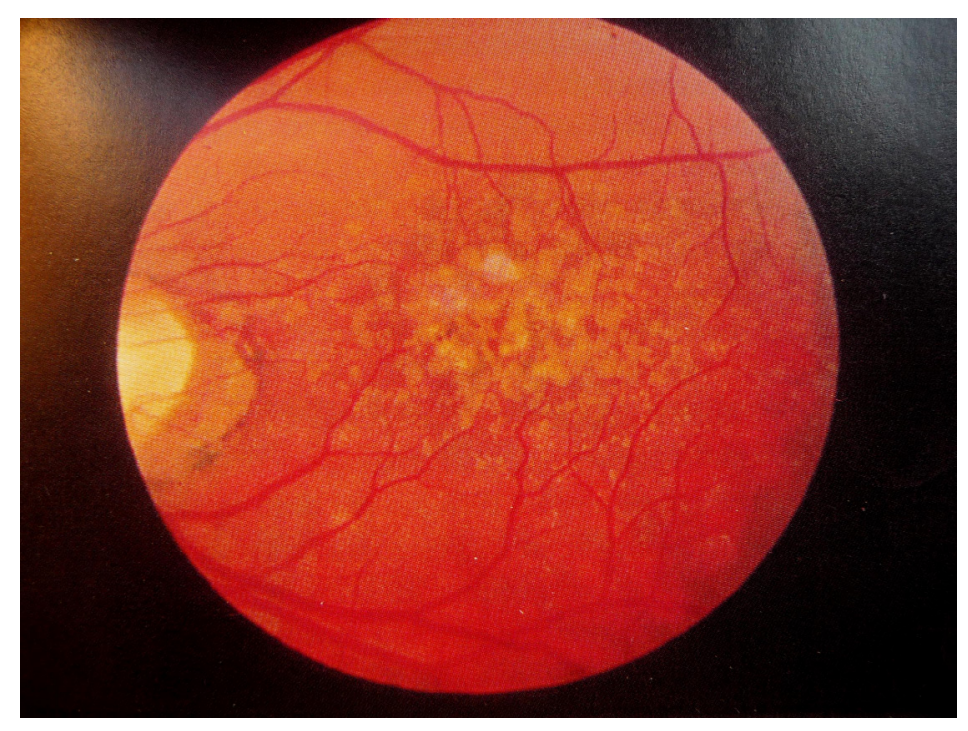

Figure 5 - Lésions photochimiques de la rétine, habituellement partiellement réversibles. Photochemical lesions of the retina usually partly reversible. 


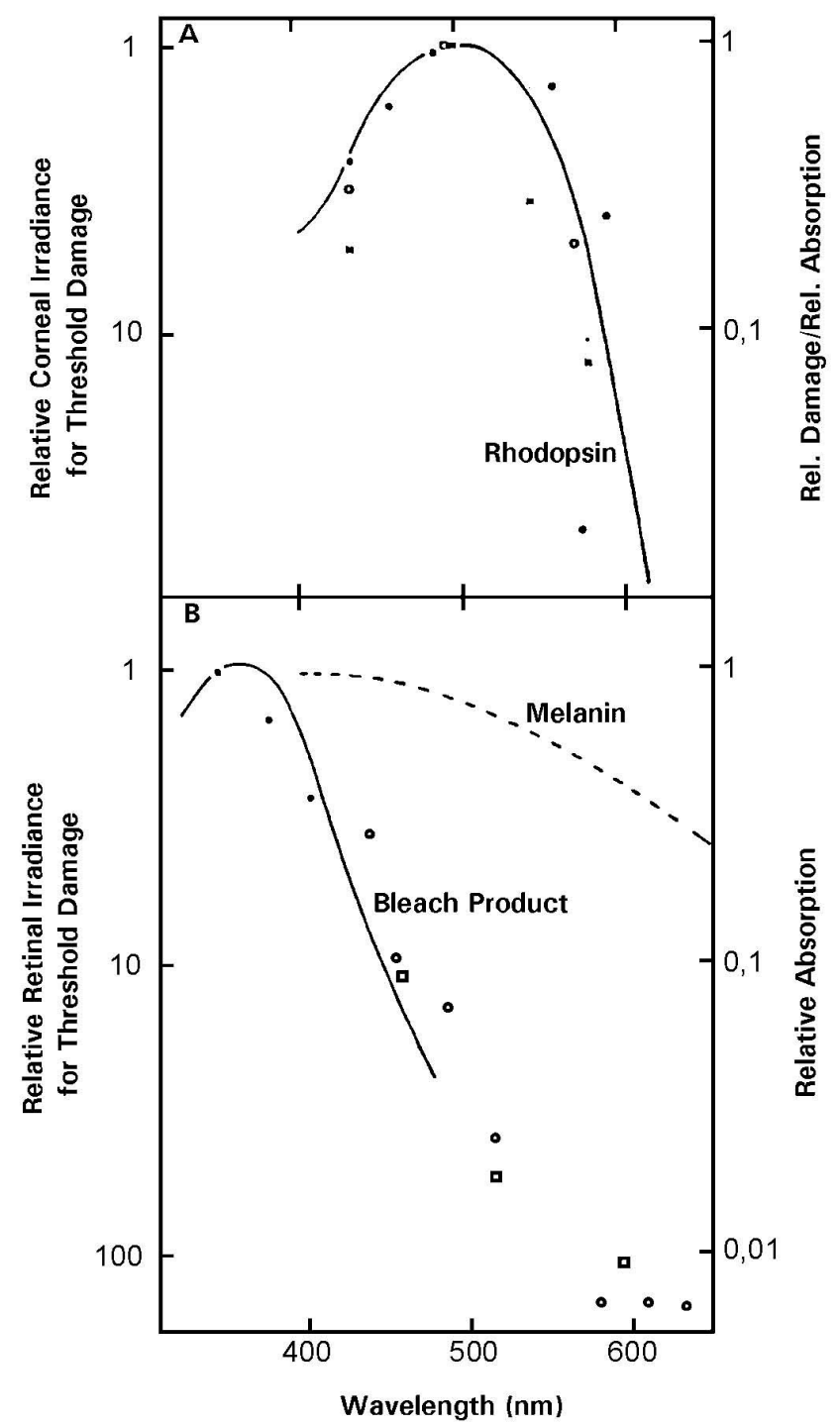

Figure 6-Propriétés d'absorption spectrique de la rhodopsine, des mélanines et leurs produits de dégradation.

Spectral absorption properties of rhodopsine, melanins, and related bleach products. 
mitochondries et même un produit secondaire du produit visuel photo-oxydé tel que rétinaldéhyde, rétinol ou 3-hydroxyrétinol.

Les figures 2, 3, 4 et 5 sont empruntées à l'ouvrage du NRPB (2002) sous la direction de Swerdlow (ICNIRP) assisté de McKinlay (CIE et ICNIRP).

\section{Fonctions spectrales pondérées du BLH et de la brûlure rétinienne}

La fonction pondérée standard, $B(\lambda)$, a été initialement établie par David Sliney puis utilisée par l'ACGIH (1992). Cette fonction est basée sur les résultats expérimentaux de Ham et al. (1976) et de Ham (1989) obtenus après observation des lésions apparues sur la rétine de singes rhésus. Cette fonction a permis d'établir des valeurs limites de seuil pour une exposition humaine (ICNIRP, 1997). Par la suite, on a développé une fonction de risque pour l'œil aphake, $A(\lambda)$ afin de prendre en compte les risques encourus par les patients ayant subi l'ablation du cristallin, sans remplacement. Notons qu'habituellement l'ablation du cristallin atteint de cataracte est suivie immédiatement du positionnement d'un cristallin artificiel contenant un filtre anti-UVA (l'implant blanc laisse passer $100 \%$ de la lumière bleue potentiellement phototoxique, l'implant jaune avec inclusion d'un pigment chromophore arrête complètement les UV, absorbe plus de $50 \%$ des radiations en dessous de $450 \mathrm{~nm}$ et de moins en moins jusqu'à $500 \mathrm{~nm}$ ).

La fonction spectrale de la brûlure thermique rétinienne se présente sous forme de courbe couvrant l'intervalle 380-1 $400 \mathrm{~nm}$ avec un pic à $440 \mathrm{~nm}$ (Fig. 7).

Les figures 6 et 8 ainsi que les tableaux I et II sont extraits du rapport à la CIE (2000) du comité technique 6-14 dirigé par Kohmoto dont Césarini a été membre de 1995 à 2002. La figure 7 est extraite du standard normatif CIE DS 009/E, EN 62471 (CIE, 2001).

Une formule permet le calcul de la valeur limite de seuil (TLV) du risque lié à la lumière bleue Le calcul du TLV pour une exposition professionnelle de l'œil aux rayonnements visible et infrarouge correspond à une exposition de 8 heures et nécessite la connaissance de la luminance énergétique spectrale $\left(\mathrm{L}_{\lambda}\right)$ et de l'éclairement énergétique total (E) de la source, mesurée à la position du travailleur. Pour protéger du risque photochimique rétinien lors des expositions chroniques à la lumière bleue, la luminance énergétique spectrale (L), pondérée par la fonction $\mathrm{B}(\lambda)$ du risque lié à la lumière bleue ne doit pas excéder $10 \mathrm{~mW} / \mathrm{cm}^{2}$. Le produit pondéré de $\mathrm{L}_{\lambda}$ et $\mathrm{B}(\lambda)$ est appelé $\mathrm{L}_{\text {bleu }}$. Lorsque la valeur 


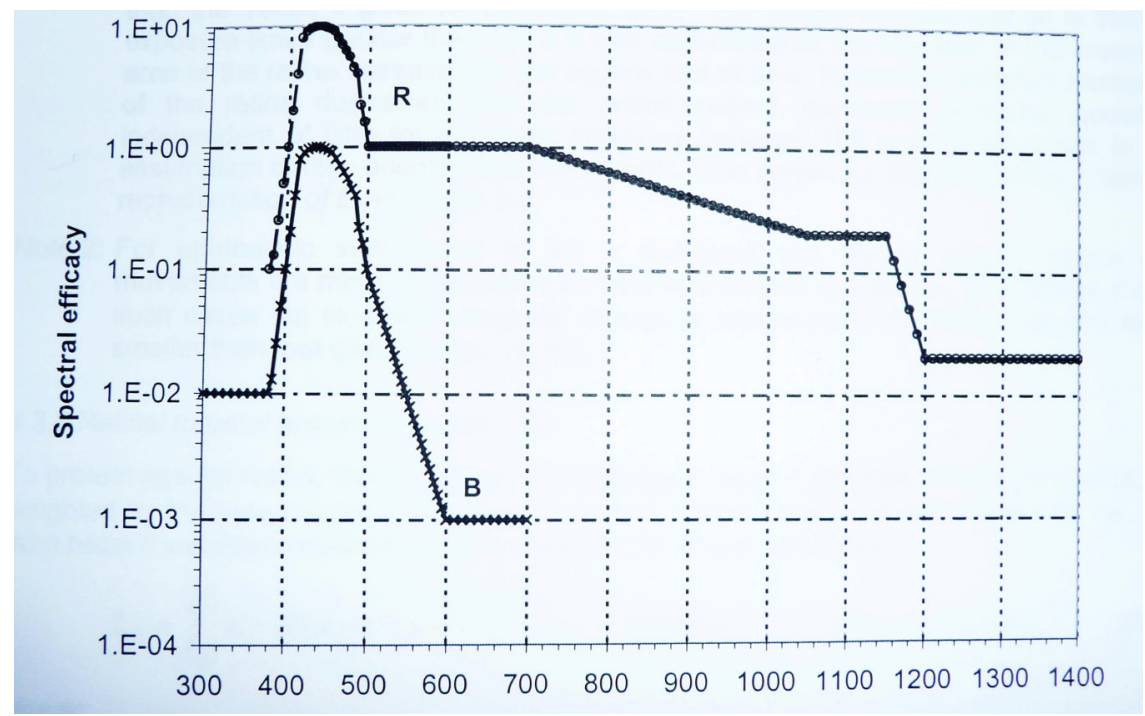

Figure 7 - Courbes d'efficacité spectrale de la brûlure thermique (R) et de la brûlure photochimique (B). Spectral efficacy of radiations to produce thermal burn $(R)$ and photochemical burn $(B)$.

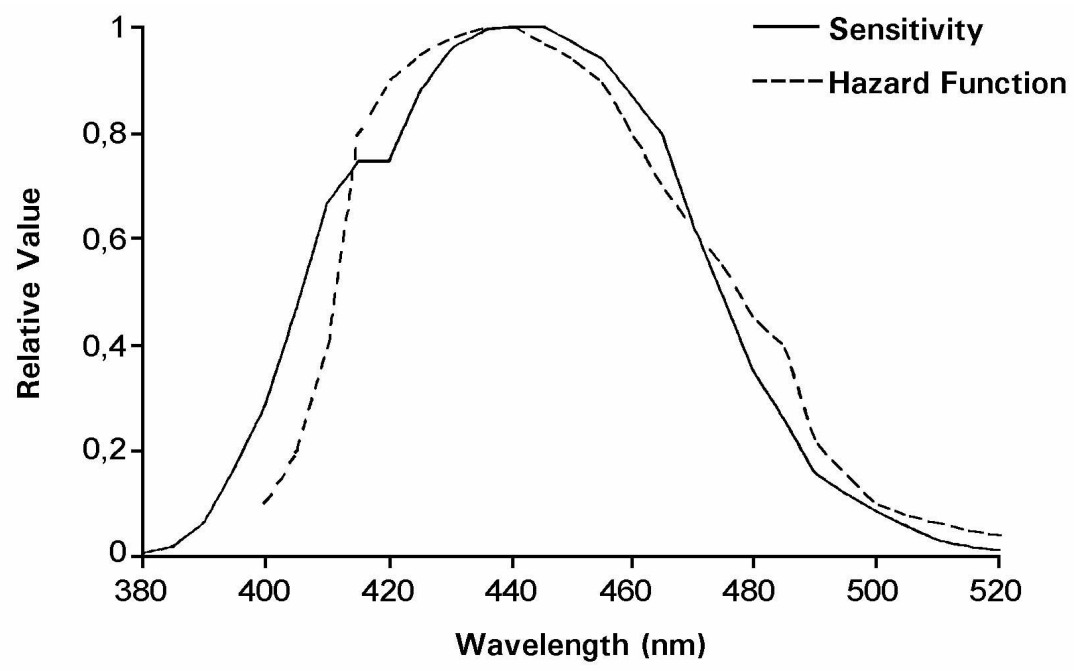

Figure 8-Comparaison du spectre d'action du risqué pour la lumière bleue avec la réponse spectrale d'un dosimètre spécifique (communication K. Komoto).

Comparison of the action spectrum for BLH with the spectral response of specific dosimeter (communication from K. Komoto). 
TABLEAU I

Sources de radiations optiques susceptibles d'induire le risque lié aux radiations bleues (BLH). Optical radiation sources likely to induce a blue light hazard.

\begin{tabular}{|c|c|c|c|c|c|}
\hline Sources & Lampe (W) & Rayonnement (W) & $\begin{array}{l}\text { Luminance énergétique } \\
\qquad\left(\mathrm{W} \mathrm{m} \mathrm{m}^{-2} \mathrm{sr}^{-1}\right)\end{array}$ & Champ de $\lambda(\mathrm{nm})$ & $\begin{array}{c}\% \text { de lumière } \\
\text { bleue }\end{array}$ \\
\hline $\begin{array}{l}\text { Rayonnement } \\
\text { solaire }\end{array}$ & $\sim$ & $1 \mathrm{~kW} \mathrm{~m}^{-2}$ & $2,2 \times 10^{7}$ & $300-2500$ & $5-10$ \\
\hline $\begin{array}{c}\text { Lampe à } \\
\text { incandescence }\end{array}$ & $10-2000$ & $9-1800$ & $10^{2}$ & $300-2500$ & $0,5-2$ \\
\hline $\begin{array}{l}\text { Tungstène } \\
\text { halogène }\end{array}$ & $500-10000$ & $450-9000$ & $10^{4}$ & $270-2500$ & $1-4$ \\
\hline Arc xénon & $500-20000$ & $250-10000$ & $10^{6-} 10^{8}$ & $250-3500$ & $6-10$ \\
\hline $\begin{array}{l}\text { Mercure haute } \\
\text { pression }\end{array}$ & $40-2000$ & $25-1200$ & $10^{3}-10^{5}$ & $300-1000$ & $8-20$ \\
\hline $\begin{array}{l}\text { Lampes à } \\
\text { métalloïdes }\end{array}$ & $100-2000$ & $80-1500$ & $10^{3}-10^{5}$ & $300-1000$ & $8-22$ \\
\hline Arc de soudure & $\sim$ & $\sim$ & $10^{8}-10^{10}$ & $200-1000$ & $5-40$ \\
\hline Référence laser & $\sim$ & $1-10 \times 10^{-3}$ & $10^{9}-10^{14}$ & $300-10000$ & $\sim$ \\
\hline
\end{tabular}

de $\mathrm{L}_{\text {bleu }}$ excède $10 \mathrm{~mW} / \mathrm{cm}^{2}$, la durée de l'exposition autorisée est définie par $\mathrm{t}_{\max }$ exprimé en secondes selon la formule :

$$
\begin{aligned}
& \sum_{400}^{700} \mathrm{~L}_{\lambda} \mathrm{B}(\lambda) \Delta \lambda \leq 10^{-2} \quad \mathrm{~W} \mathrm{~cm}^{-2} \mathrm{sr}^{-1} \quad\left(t>10^{4} \mathrm{~s}\right) \\
& \sum_{400}^{700} \mathrm{~L}_{\lambda} t \mathrm{~B}(\lambda) \Delta \lambda \leq 100 \quad \mathrm{~J} \mathrm{~cm}^{-2} \mathrm{sr}^{-1} \quad\left(t \leq 10^{4} \mathrm{~s}\right) \\
& t_{\max }=100\left(\mathrm{~J} \mathrm{~cm}^{-2} \mathrm{sr}^{-1}\right) / \mathrm{L}_{\text {blue }} \text { ou } 10^{6}\left(\mathrm{~J} \mathrm{~m}^{-2} \mathrm{sr}^{-1}\right) / \mathrm{L}_{\text {blue }}
\end{aligned}
$$

\section{Sources de radiations optiques susceptibles d'induire le BLH}

Des sources de radiation variées, y compris le soleil, sont susceptibles d'être regardées directement. Cependant, la vision sécurisée de ces sources intenses dépend de la durée de l'observation. Certaines sources sont particulièrement riches en radiations de la région de la lumière bleue. Le tableau I exprime le pourcentage de lumière bleue ainsi que la luminance énergétique de quelques sources naturelle et artificielles. Le tableau II montre les valeurs de luminance énergétique pour la composante bleue de quelques sources lumineuses. 
TABLEAU II

Luminance énergétique de la lumière bleue de différentes sources. Radiance of the blue light from different sources.

\begin{tabular}{ll}
\hline Sources de lumière & Luminance énergétique $\left(\mathrm{W} \mathrm{cm}^{-2}\right)$ \\
\hline Soleil & 62,4 \\
Arc de soudure & 10,5 \\
\hline Lampe à incandescence $(100 \mathrm{~W})$ & 0,00102 \\
Lampe à incandescence $(57 \mathrm{~W})$ & 0,000865 \\
Lampe à fluorescence & 0,00036 \\
Valeur seuil pour une exposition de plus de $10^{4}$ secondes & 0,01 \\
\hline
\end{tabular}

\section{Appareils de mesure destinés a l'évaluation optique par rapport au BLH}

Grâce à des filtres appropriés, on a pu concevoir un appareillage dont la sensibilité spectrale relative coïncide avec la fonction $\mathrm{B}(\lambda)$ (Fig. 8). Les normes AFNOR $(2004,2006)$ précisent le mesurage et le formatage concernant les rayonnements visibles et infrarouges émis par des sources artificielles sur les lieux de travail.

\section{Sensibilité à la lumière}

Dans le cadre des communautés européennes (EU, 2006), le comité scientifique sur les risques pour la santé, récemment identifiés et émergents (SCENIHR), a été saisi des problèmes de sensibilité de l'homme au rayonnement visible dans le cadre de la promotion de l'usage de sources lumineuses économiques telles que les lampes compactes fluorescentes (CFLs) et la disparition probable des lampes à incandescence. Leurs effets cutanés et oculaires ont été analysés et leur impact sur la santé évalués par un groupe de travail composé de 3 membres du SCENIHR et de 4 membres extérieurs, scientifiques, spécialistes des rayonnements visibles et ultraviolets, qui a remis en septembre 2008 ses conclusions (SCENIHR, 2008).

«Le SCENIHR a examiné les CFLs pour 3 caractéristiques essentielles : le clignotement, les champs électromagnétiques et l'émission de lumière UV et bleue qui sont susceptibles de déclencher certains symptômes liés à des pathologies ». En raison du manque de données sur les CFLs, les données existantes pour les tubes fluorescents traditionnels ont été extrapolées en situations où les CFLs sont utilisées.

« De toutes les propriétés des CFLS, seul le rayonnement UV/lumière bleue a été identifié comme un facteur de risque potentiel pour aggraver les symptômes de 
RISQUES OCULAIRES DU RAYONNEMENT BLEU

sensibilité à la lumière chez certains patients souffrant de dermatite actinique chronique et d'urticaire solaire ».

Le comité a attiré l'attention sur le fait que des CFLs à une seule enveloppe émettent des UVB et des traces d'UVC, ce qui, dans certaines conditions extrêmes telles que les expositions prolongées à proximité de ces CFLs, induit des expositions UV proches des limites établies pour les dommages cutanés et oculaires (rétiniens) chez les travailleurs postés.

Le nombre de patients sensibles à la lumière serait de 250000 dans l'Union européenne.

«Le comité a préconisé l'utilisation d'une double enveloppe pour les CFLs et technologies similaires afin de réduire ou supprimer entièrement les risques pour les travailleurs postés ainsi que le risque d'aggravation des symptômes chez les sujets sensibles à la lumière ».

\section{Dégénérescence maculaire liée à l’âge (DMLA)}

La macula est la partie centrale de la rétine riche en cellules en cônes, responsable du sens morphoscopique (acuité visuelle) et du sens chromatique de la vision précise et colorée. La DMLA est une condition pathologique spécifique de la macula entraînant une perte progressive de l'acuité visuelle. La macula représente la source majeure de cécité légale dans les sociétés évoluées (WHO, 1994). C'est une condition multifactorielle où le vieillissement et les facteurs génétiques jouent un rôle essentiel. Cliniquement, on distingue une forme d'apparition précoce (maculopathie liée à l'âge ou MLA) et une forme d'apparition tardive (dégénérescence maculaire liée à l'âge ou DMLA). Dans les deux cas, des changements dégénératifs (druses) de la membrane basale siégeant à l'interface de la rétine et de la choroïde sont observés habituellement et sont nettement liés à l'âge. Il a été suggéré que les UVA et la lumière bleue contribuent à la dégénérescence maculaire (Young, 1981). La prévalence de la DMLA est diminuée lorsqu'il y a opacification du cristallin, en particulier en cas de cataracte nucléaire. Par ailleurs, les études portant sur les pécheurs et les constructeurs de barrages ont montré une association nette entre la DMLA et l'exposition à la lumière sur une période de vingt ans. Par ailleurs, le port de lunettes était inversement associé à l'augmentation de la pigmentation rétinienne et des druses rétiniens. D'autres études épidémiologiques sont plus convaincantes, montrant une association entre DMLA exsudative et la DMLA tardive avec la quantité d'expositions lors des loisirs chez l'homme et chez la femme. Une association positive a été trouvée également en Australie entre la couleur bleue des yeux, l'hypersensibilité cutanée au soleil, les coups de soleil fréquents, les cancers 
cutanés et la survenue tardive d'une DMLA. Une agression chronique par les radicaux libres induits par la lumière visible parât être un mécanisme important dans l'induction de la DMLA. Cette hypothèse semble confirmée par la stabilisation des lésions après prise quotidienne d'antioxydants (sélénium) par avoie orale (Césarini, 2004).

\section{Protection oculaire conventionnelle}

Plusieurs types de protection oculaire sont commercialisés. Ces types sont généralement classés selon une des trois catégories: lunettes antisolaires, protection générale de l'œil dans l'industrie et protecteurs oculaires contre les lasers.

(a) Lunettes antisolaires : les lunettes antisolaires achetées par les consommateurs sont avant tout choisies sur des considérations de mode et d'aspect esthétique, le design des formes et la couleur étant considérés comme plus important que leurs propriétés optiques. De très nombreuses lunettes ne respectent pas les standards nationaux ou ISO pour les lunettes antisolaires. Par conséquent, en général les lunettes colorées ne peuvent pas être utilisées pour protéger l'œil humain des sources dangereuses telles que l'arc de soudure, lampes xénon, vision directe du soleil ou de sa réflexion sur les surfaces horizontales telles que neige, glace, écume de mer, etc. Le marquage $\mathrm{CE}$, obligatoire, distingue quatre niveaux de protection : les niveaux 2 et 3 sont habituellement suffisants pour assurer une bonne protection; le niveau 4, utilisé en montagne, sur glacier, est impropre à la conduite automobile. Il est également important de protéger les enfants dont le cristallin clair n'exerce pas sa fonction de filtre pour les UVA dont $75 \%$ sont transmis à la rétine avant 10 ans alors que moins de $10 \%$ seulement sont transmis au-delà de 25 ans.

(b) Les protecteurs oculaires généralement utilisés dans l'industrie sont fabriqués pour protéger le travailleur non seulement contre des sources lumineuses intenses mais aussi pour protéger contre d'éventuels objets volants. La protection des yeux des travailleurs spécialisés dans les fours et toutes sources de radiations thermiques constitue une sub-catégorie spécifique. Pour les protecteurs à usage industriel on distingue trois catégories de formes : (1) type lunette de vue, (2) type goggle, (3) casque ou protection tenue à la main. Pour ces trois types les propriétés optiques de transmission sont considérées comme des spécificités importantes et font par conséquent l'objet de standards appropriés.

(c) Les protecteurs oculaires vis-à-vis des lasers nécessite des spécifications techniques originales car les lasers présentent des risques extrêmement sérieux. La spécificité des longueurs d'onde et la densité optique sont des facteurs 
critiques dans le choix d'une protection oculaire appropriée. Néanmoins, de nombreuses sources émettant dans des vastes longueurs d'onde, doivent être considérées quant au risque de BLH. Ce risque est déjà pris en considération dans les standards de sécurité des lasers.

On trouvera en bas de page quelques normes ISO concernant la protection oculaire $^{2}$. Dans ces standards, la protection contre BLH n'est pas toujours spécifiée.

\section{Conclusion}

Les risques de lésions oculaires liés à la lumière bleue sont bien réels. Les lésions immédiates ou chroniques peuvent être évitées grâce aux mesures appropriées, notamment par le port de lunettes adaptées aux conditions de travail, à la l'exposition à la lumière solaire.

Il apparaît important d'exercer un contrôle strict de l'émission des éclairages artificiels, notamment en accompagnant leur utilisation de recommandations élémentaires :

- éviter de regarder fixement les sources lumineuses ;

- mettre en place les dispositifs d'éclairage indirect ou filtrer les sources halogènes ;

- maintenir à distance raisonnable les sources CFL ou LED ;

- être vigilent vis-à-vis des sources de multiples LED disposées en panneaux ;

- être vigilent vis-à-vis de sources bénéficiant de publicités assurant des bénéfices pour la santé telles que «faire entrer le soleil», «faire le plein d'énergie », « la vie au naturel »...

\section{RÉFÉRENCES}

AFE (1993) Association française de l'éclairage, Les radiations optiques en médecine. Éditions LUX, Paris.

ACGIH (1992) American Conference of Governmental Industrial Hygienists, Documentation for the threshold limit values, 4th edition, Cincinnati, OH, USA.

AFNOR (2004) Norme NF EN 13032-1, Mesure et présentation des données photométriques des lampes et des luminaires - Partie 1 : Mesurage et format de données.

AFNOR (2006) Norme NF EN 14255-2, Mesurage et évaluation de l'exposition des personnes aux rayonnements optiques incohérents - Partie 2 : Rayonnements visibles et infrarouges émis par des sources artificielles sur les lieux de travail.

2 ISO 4850-1979 : protection oculaire personnelle pour la soudure et techniques reliées : filtres, qualité de transmission et utilisation; ISO 4851-1979: protection oculaire personnelle: filtres ultraviolets, qualité de transmission et utilisation; ISO 4849-1981 : protection personnelle des yeux : spécification ; ISO 4854-1981 : protection oculaire personnelle : méthodes de test optiques. 


\section{J.-P. CÉSARINI}

Césarini J.P. (2004) Le Sélénium: Actualités, Collection pathologie science. John Libbey Eurotext, Paris, pp. 70-71.

CIE (2000) CIE TC6-14 report: blue light photochemical retinal hazard published in the CIE collection in Photobiology and Photochemistry 138/1.

CIE (2001) CIE Standard, Photobiological safety of lamps and lamp systems, CIE DS 009.2/E: 2001 and EN 62471

Cronley-Dillon J., Rosen E.S., Marshall J.E. (1986) Hazards of light: myths and realities. Pergamon Press, London.

EU (2006) European Union O.J. Directive 2006/25/EC of the European Parliament and of the Council. 05-2006 "On the minimum health and safety requirements regarding the exposure of workers to risks arising from physical agents (artificial optical radiation)" (19th individual Directive within the meaning of Article 16(1) of Directive 89/391/EEC)".

Ham W.T., Mueller H.A. Sliney D.H. (1976) Retinal sensitivity to damage from short wavelength light, Nature 260, 153-155.

Ham W.T. (1989) The photopathology and nature of the blue-light and near-UV retinal lesion produced by lasers and other optical sources, In: Laser Applications in Medicine and Biology, edited by M.L. Wolbarsht, New-York Publishing Corp.

ICNIRP (1997) International Commission on Non-Ionizing Radiation Protection, Guidelines on limits of exposures for broadband incoherent optical radiation $(0.38$ to $3 \mu \mathrm{m})$, Health Phys. $\mathbf{7 3}$, 539-555; www.icnirp.net/downloads.htm.

Marshall J. (1985) Radiation and the ageing eye, Ophthalmic Physiol. Opt. 5, 241-263.

NRPB (2002) National Radiological Protection Board, Health effects from ultraviolet radiation. OX11 ORQ, UK, ISBN 0-85951-475-7. www.nrpb.org.

SCENIHR (2008) Scientific Committee on Emerging and Newly-Identified Health Risks, Scientific opinion on light sensitivity, septembre 2008, EC-Directorate-General for Heath \& Consumers.

WHO (1994) Environmental Health Criteria 160: Ultraviolet Radiation, Ch. 10, Human studies: the eye; pp. 181-207.

Young R.W. (1981) A theory of central retinal disease. In: New Directions in Ophthalmic Research, edited by M.L. Sears, Yale University Press, New Haven, Connecticut, pp. 237-270. 